\title{
Traumatic Pneumolabyrinth: A Case Secondary to Palm Frond Penetration and a Review of the Literature
}

\author{
Jennifer Ha, Peter Gochee, Peter Santa Maria and Philip Grey \\ Department of Otolaryngology Head \& Neck Surgery, Sir Charles Gairdner Hospital, Hospital Avenue, \\ Nedlands, Western Australia 6009, Australia \\ Correspondence should be addressed to: Jennifer Ha; Jennifer_fong_ha@yahoo.com.au
}

Received 2 March 2013; Accepted 3 April 2013; Published 28 August 2013

Academic Editor: Yíldírím Ahmet Bayazít

Copyright @ 2013 Jennifer Ha, Peter Gochee, Peter Santa Maria and Philip Grey. Distributed under Creative Commons CC-BY 3.0

\begin{abstract}
We describe a case of a 57-year-old man with severe vertigo, associated with nausea and vomiting, left-sided bloody otorrhea, hearing loss, and tinnitus, following a traumatic puncture by a palm frond. We present the diagnostic dilemma associated with the traumatic pneumolabyrinth, the potential for perilymphatic fistula, and the controversies in management.
\end{abstract}

Keywords: Perilymphatic fistula, traumatic, pneumolabyrinth, management, diagnosis, systematic review.

\section{Introduction}

Pneumolabyrinth (PL) is air trapping in the vestibulocochlear system due to abnormal communication between the inner ear and its surrounding structures. This was first introduced by Mafee et al. (1). Perilymphatic fistula (PLF) is an uncommon clinical manifestation, with only 29 reported cases in the literature $(2$, $3)$. It was first described by Escat in 1897, and it was scarcely reported for decades until the $1960 \mathrm{~s}$ as a complication of otosclerosis surgery. In 1968, Fee and colleagues reported the first non-iatrogenic PLF $(4,5)$. It has been described following head traumas, temporal bone fractures, barotrauma, iatrogenic injuries, cholesteatoma, and middle ear neoplasms $(2,5-7)$.

\section{Methods}

A literature review was performed with the MEDLINE database with the keywords "traumatic perilymphatic fistula."

\section{Case Presentation}

A 57-year-old man presented with severe vertigo, associated with nausea and vomiting, as well as left-sided otalgia and bloody otorrhea, hearing loss (HL), and tinnitus, following a traumatic puncture by a palm frond (Yucca elephantipes). The injury was sustained while the patient was walking backwards and turned suddenly when the tip of the frond entered his ear.

He was referred to the Sir Charles Gairdner Hospital, Otolaryngology Department, Perth, Australia, 24 hours later with

Cite this Article as: Jennifer Ha, Peter Gochee, Peter Santa Maria and Philip Grey (2013), "Traumatic Pneumolabyrinth: A Case Secondary to Palm Frond Penetration and a Review of the Literature," International Journal of Case Reports in Medicine, Vol. 2013 (2013), Article ID 803147, DOI: $10.5171 / 2013.803147$ 
persistent symptoms. Clinically, he had nystagmus away from the affected side. Otoscopy revealed a subtotal perforation with blood in the external auditory meatus. Tuning fork test was consistent with a conductive HL on the affected side. Audiometry revealed a mild mixed HL with a $15 \mathrm{~dB}$ conductive component (Figure 1). The high resolution computed tomography (HRCT) of his temporal bone revealed pneumolabyrinth in the vestibule and ampulla of the lateral semicircular canal (Figure 2). There were neither fracture lines seen, nor disruption to the ossicles.

FREQUENCY IN HERTZ

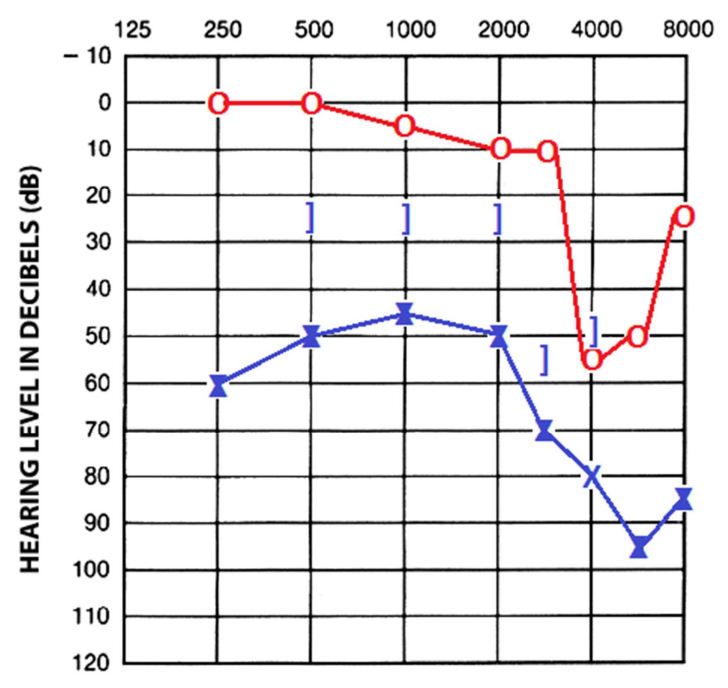

Figure 1: Pre-Operative Audiogram

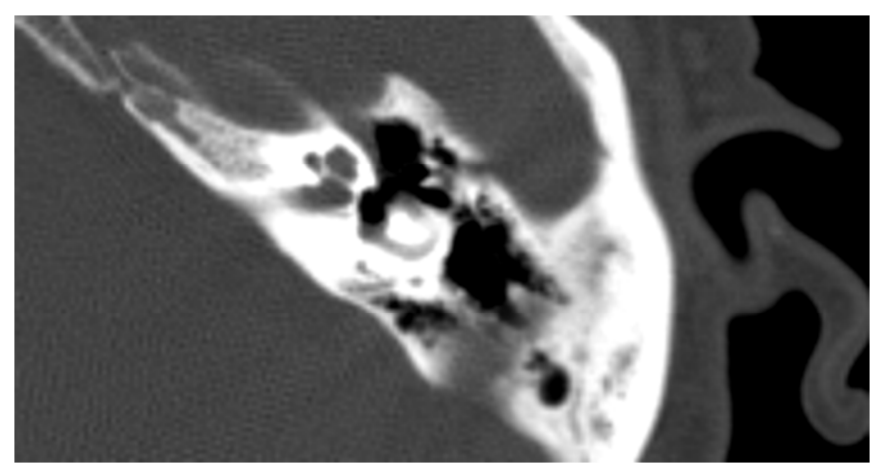

Figure 2: HRCT of the Temporal Bone Revealing Pneumolabyrinth in the Ampulla of the Lateral Semicircular Canal

Due to the persistence of his symptoms, he underwent an exploratory tympanotomy. There was an approximately $40 \%$ perforation of the posterior portion of the tympanic membrane. After an elevation of a tympanomeatal flap, a large traumatic cochleostomy just inferior and slightly anterior to the round window was observed. The stapes suprastructure, incudostapedial joint, and malleus were intact. Ossicular continuity was confirmed.
The palm frond had not been retained. Temporalis muscle was applied over the traumatic puncture, and temporalis fascia was used to repair his tympanic perforation.

Postoperatively, he was treated with strict bed rest for 48 hours, regular stool softener, analgesics, and antiemetics. His symptoms improved dramatically postoperatively. He was discharged on the 
fourth postoperative day. His postoperative audiogram showed a moderate sensorineural HL (Figure 3). It is likely that this did not progress to a profound loss due to the rapid identification and surgical management of this patient. It is presumed that the course of the frond was directed posteriorly, which would explain its entrance into the vestibule. His middle ear packing was removed four weeks postoperatively and revealed a healed tympanic membrane.

FREQUENCY IN HERTZ

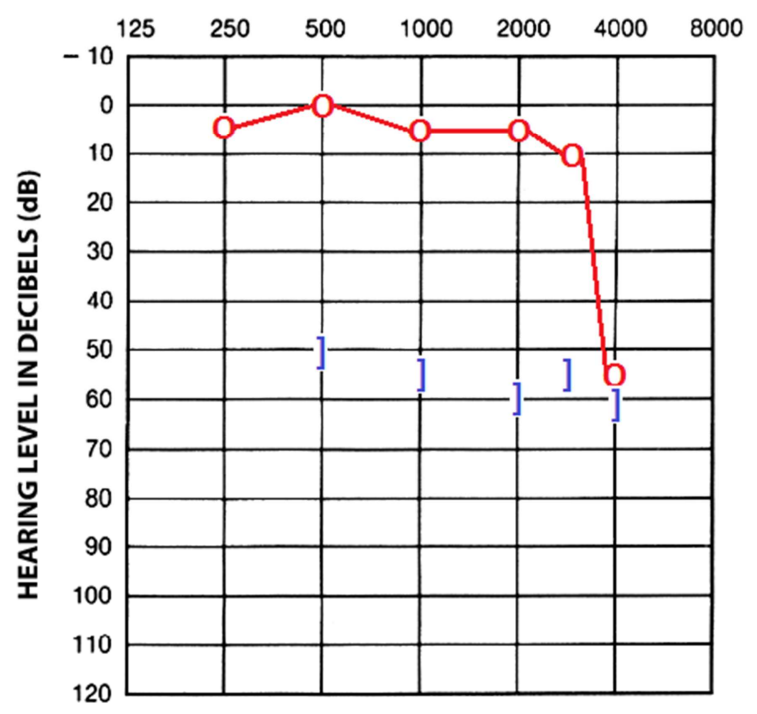

Figure 3: Post-Operative Audiogram

\section{Discussion}

There were 29 cases of PLF reported in the literature, which is presented in Table 1. Four cases reported by Xie et al. were in
Chinese, a case by Baron et al. in French, and Saito et al. and Uemura et al. each reported a case in Japanese that we were not able to assess due to language limitations of the authors. 
Table 1: Reported Traumatic PLF in the Literature

\begin{tabular}{|c|c|c|c|}
\hline Etiology & Author & Treatment & Repair \\
\hline \multirow[t]{8}{*}{ Temporal Bone Fracture } & Lipkin $^{3}$ & Surgery & Fat from ear lobule \\
\hline & Lyos $^{33}$ & Surgery & $\begin{array}{l}\text { Temporalis fascia \& } \\
\text { muscle }\end{array}$ \\
\hline & Lyos $^{33}$ & Surgery & $\begin{array}{l}\text { Temporalis fascia \& } \\
\text { muscle }\end{array}$ \\
\hline & Yanagihara 21 & Surgery & Tragal perichondrium \\
\hline & Gross 16 & Conservative & \\
\hline & Prisman $^{19}$ & Conservative & \\
\hline & Prisman 19 & Conservative & \\
\hline & Prisman $^{19}$ & Conservative & \\
\hline Lightning & Sun & Surgery & $\begin{array}{l}\text { Temporalis fascia \& } \\
\text { muscle }\end{array}$ \\
\hline \multirow[t]{2}{*}{ Slap } & Yangihara 21 & Surgery & Tragal perichondrium \\
\hline & $\mathrm{Lo}^{11}$ & Surgery & Tragal perichondrium \\
\hline $\begin{array}{cc}\text { Penetrating injury } \\
-\quad \text { Knitting needle }\end{array}$ & Yanagihara $^{21}$ & Surgery & Tragal perichondrium \\
\hline$-\quad$ Tree branch & $\mathrm{LaO}^{3}$ & Conservative & \\
\hline \multirow[t]{4}{*}{ - $\quad$ Ear pick } & Tsubota $^{34}$ & Surgery & Temporalis fascia \\
\hline & Uemura* & Surgery & \\
\hline & Saito* & Surgery & \\
\hline & Yamasoba $^{24}$ & Surgery & Tragal perichondrium \\
\hline - $\quad$ Paintbrush & Rajan $^{18}$ & Surgery & Connective tissue \\
\hline$-\quad$ Cotton bud & Herman 12 & Conservative & \\
\hline \multirow[t]{5}{*}{ Blunt injury } & Sarac $^{3}$ & Surgery & Fat \\
\hline & Lyos $^{33}$ & Surgery & $\begin{array}{l}\text { Temporalis fascia \& } \\
\text { muscle }\end{array}$ \\
\hline & De Negreiros $^{6}$ & Conservative & \\
\hline & Nurre 32 & Conservative & \\
\hline & Woo $^{4}$ & Conservative & \\
\hline
\end{tabular}

\section{Etiology}

Traumatic PLF can be congenital or acquired. In the congenital category, it may be associated with or without other cranial anomalies (4). PLF was first commonly recognised as a pathologic entity following a complication of stapedectomy $(4,6)$. Noniatrogenic can be classified into traumatic, erosive, or idiopathic $(6,8)$.

\section{Pathophysiology}

Traumatic PLF may be a result of implosive trauma, secondary to an external force. These include barometric pressure change, compressive trauma of the ear, and Valsalva's maneuver. This leads to pressure changes in the middle ear space, exerting external force on the oval and round window membranes, fissula ante fenestram, microfissures, and Hyrtl's fissure $(2,5,6)$.

Explosive trauma has also been found to be a cause. This results from an internal change induced by increased cerebrospinal fluid pressure from lifting, straining, coughing, or sneezing, resulting in intracochlear membrane damage (4-6). Erosive destruction of inner and middle ear cavity structures by cholesteatoma, chronic otitis media, otologic malignancy, or surgery are other causes described (2).

The symptoms are thought to relate to the effect of the presence of air on the inner ear membrane potential (2). Entry of air through an apical opening increases the threshold of action potentials of the auditory nerve (2). The initial HL is caused by disruption of the cochlear microphonics and action potential generation mechanism rather than strial dysfunction (2). It has 
been demonstrated that poorer prognosis is associated with oval window fistula compared to that of the round window (2). HL was induced in animal models with the introduction of air into the inner ear system. This disturbed the propagation of the traveling wave of the basilar membrane $(5,7)$. The magnitude of HL depends on the location of air in the inner ear. Air in the scala vestibuli has a greater impact on HL and is often irreversible, compared with the scala tympani which causes less HL and is often reversible with removal of air (7). The worst outcome reported is air in the cochlea compared to that isolated in the vestibule (8). Leakage of perilymph may induce relatively high endolymphatic pressures, which agitates the membranous labyrinth causing vertigo or unsteadiness (5).

\section{Diagnosis}

There is no objective means to establish the diagnosis. The most important diagnostic method is history and physical examination. The symptoms are varied and include progressive or fluctuating hearing loss, tinnitus, aural fullness, and vertigo $(2$, $6,8,9)$. Vestibular symptoms are reported in $80 \%$ of patients with PLF, and $64 \%$ are associated with a tympanic membrane perforation (6). Positive fistula test (positive pressure to the tympanic membrane to induce nystagmus), Tullio phenomenon (vertigo/loss of balance after exposure to a $95 \mathrm{~dB}$ tone burst at $500 \mathrm{~Hz}$ for $<3$ seconds), and Hennebert's sign all have a low sensitivity (5). However, some authors advocated that the fistula test should be avoided due to the presumed risk for further cochlear injury and worsening HL (2).

Audiometry may show a conductive loss as presence of air in cochlea results in an impedance mismatch with reduced sound transmission through the perilymph, or secondary from a hemotympanum (10).

$\beta$-trace protein or $\beta 2$-transferrin detection in the middle ear fluid has been reported to be useful, but the sensitivity is low $(5,8)$. Electrocochleography has been reported as a measure of the electrical activity of cochlea (11). Its use intra-operatively can indicate changes in cochlea function during surgery and detect PLF when PLF leakage is not obvious by showing an increased summation potential/action potential ratio (5). However, this is not also a sensitive test for PLF (4). Transtympanic endoscopy has been reported to be a useful office procedure that provides excellent middle ear content exposure and is minimally invasive $(3,12)$.

Magnetic resonance imaging is not appropriate as it is difficult to distinguish air from the otic cortical bone adjacent to air (neither air nor cortical bone produces signal) (5). Temporal bone HRCT is the imaging modality of choice in PL, but sometimes exploratory tympanotomy may be required for the diagnosis $(5,7)$.

Intraoperative diagnosis is based on the observation of clear fluid pooling at the oval or round window, or from a patent fissula ante fenestrum or fissula post fenestrum, which may be spontaneous or enhanced by elevation of intrathoracic pressure (13). Air bubbles have also been described intra-operatively (8). A stringent criterion suggested by Myer et al. is the presence of clear fluid that accumulates rapidly after suctioning, with no surrounding fluid accumulation in the operative field (3). However, any fluid in the middle ear tends to pool in the most dependent area, including the oval and round windows (13). In addition, it has been shown that injected anesthetic agents accumulate in both window niches and may resemble PLF $(3,5,13)$. Transudates from surgical incisions and mucosal injury, inherent in raising a tympanomeatal flap, may also pool in the middle ear (13). Intraoperative maneuvers like forced Valsalva, bilateral jugular vein compression, and Trendelenburg position may be helpful in discovering subtle leaks (6). The oil-on-water technique has also been reported to be useful: clear fluid droplet trapped under a layer of mineral oil, which increases in size when intracranial pressure is elevated (3). The layer of mineral oil enhances the direct visualisation of perilymph fluid and 
enhances the recognition of the location of PLF (3).

\section{Management}

The management of PL has remained controversial. Conservative management includes strict bed rest, 30 degrees head elevation, regular use of stool softener and antiemetics, and the avoidance of straining such as via Valsalva's manoeuvre $(1,2,9)$. Exploratory tympanotomy is recommended in patients with aggravation and/or persistence of vestibular symptoms, persistent discharge, and progressive or fluctuating HL $(6-8,14)$.

A variety of materials have been described in the literature for PLF repair including fat, fascia, muscle, and perichondrium, as well as adjunctive use of gelfoam, fibrin glue, fibrinogen, and thrombin grafts. Management of a displaced stapes may require reconstruction with or without its removal (2). When the stapes is not depressed into the vestibule, sealing of the PLF followed by ossicular chain reconstruction may be performed without addition risk to the inner ear (15). However, when stapes is depressed, it is important to consider if surgery is necessary and if the stapes should be removed as additional manipulation may cause additional damage to the inner ear (15).

Postoperative instructions include no significant bending, lifting, or straining period of at least 6 weeks, as this often results in a recurrent PLF (9).

Outcome measures are difficult to analyse due to the range of complaints at initial presentation. In addition, surgical exploration and correction of PL has been inconsistent at improving HL. The predictive factors of hearing improvement include interval until surgery, bone conduction hearing level at the onset of disease, and existence of a stapes injury (1, 15). Early intervention leads to a more rapid resolution of symptoms and preservation of existing auditory function $(9,14)$. Increased risk of permanent auditory loss is associated with delay closure of PLF, usually more than two weeks $(6,9,10)$. The prognosis is worse for vestibular symptoms (1). PLFs persisting for greater than seven days have an increased risk of developing meningitis (6).

\section{Conclusion}

This is an unusual mechanism of injury that has the potential of significant morbidity if not managed appropriately in a timely fashion. Whilst there is no consensus on the management of PL, it should be tailored to the etiology of PL and patient symptomatology, based on the clinician's clinical judgment. The relative safety of exploratory tympanometry balanced with incapacitating manifestation of PL and its associated risk mandates early surgical exploration.

\section{References}

1. Adil, E. A., Choudhary, A. K., Moser, K. W. \& Ghossaini, S. N. (2011). "Vestibular Pneumolabyrinth: Why Assessment with Temporal Bone Computed Tomography Utilizing Dynamic Focal Spot Mode Is Important for the Diagnosis," Emergency Radiology. 18:43-5.

2. Lao, W. W. \& Niparko, J. K. (2007). "Assessment of Changes in Cochlear Function with Pneumolabyrinth after Middle Ear Trauma," Otology \& Neurotology. 28:1013-7.

3. Todd, N. W. \& Jackson, R. T. (1995). "Oil-On-Water: Proposed Method for Intraoperative Identification of Perilymphatic Fistula," American Journal of Otology. 16(4):539-42.

4. Glasscock, M. E., Hart, M. J., Bhansali, B. A. \& Rosdeutscher, J. D. (1992). "Traumatic Perilymphatic Fistula: How Long Can Symptoms Persist? A FollowUp Report," American Journal of Otology. 13(4):333-8.

5. Lo, S. H., Huang, Y. C. \& Wang, P. C. (2003). "Pneumolabyrinth Associated with Perilymph Fistula," Chang Gung Medical Journal. 26(9):690-3. 
6. Davis, R. E. (1992). "Diagnosis and Management of Perilymph Fistula: The University of North Carolina Approach," Otology \& Neurotology. 13(1):85-9.

7. Gross, M., Ben-Yakoov, A., Goldfarb, A. \& Eliashar, R. (2002). "Pneumolabyrinth: An Unusual Finding in a Temporal Bone Fracture," International Journal of Pediatric Otorhinolaryngology. 67:553-5.

8. Prisman, E., Ramsden, J. D., Blaser, S. \& Papsin, B. (2011). "Traumatic Perilymphatic Fistula with Pneumolabyrinth: Diagnosis and Management," The Laryngoscope. 121:856-9.

9. Weider, D. J. (1992). "Treatment and Management of Perilymphatic Fistula: A New Hampshire Experience," Otology \& Neurotology. 13(2):158-66.

10. Hazell, J. W. P., Fraser, J. G. \& Robinson, P. J. (1992). "Positional Audiometry in the Diagnosis of Perilymphatic Fistula," American Journal of Otology. 13(3):263-9.

11. Gibson, W. P. (1992). "Electrocochleography in the Diagnosis of Perilymphatic Fistula: Intraoperative Observations and Assessment of a New Diagnostic Office Procedure," American Journal of Otology. 13(2):146-51.

12. Poe, D. S., Robeiz, E. E. \& Pankratov, M. M. (1992). "Evaluation of Perilymphatic Fistulas by Middle Ear Endoscopy,"American Journal of Otology. 13(6):529-33.

13. Poe, D. S. \& Bottrill, I. D. (1994). "Comparison of Endoscopic and Surgical Explorations for Perilymphatic Fistulas," American Journal of Otology. 15(6):735-8.

14. Lyos, A. T., Marsh, M. A., Jenkins, H. A. \& Coker, N. J. (1995). "Progressive Hearing Loss after Transverse Temporal Bone Fracture," Archives of
Otolaryngology- Head \& Neck Surgery. 121:795-9.

15. Yamasoba, T., Amagai, N. \& Karino, S. (2003). "Traumatic Luxation of the Stapes into the Vestibule," Otolaryngology - Head and Neck Surgery. 129:287-90. 